\title{
Avaliação da capacidade de rebrotamento pós-distúrbio das plantas lenhosas típicas dos campos rupestres
}

\author{
Bárbara de Carvalho Barbosa ${ }^{\bowtie 1,2}$; Viviane Siqueira Cappi ${ }^{3}$; Sérvio Pontes Ribeiro ${ }^{3,4}$ \\ \& G. WILSON FERNANDES ${ }^{2}$
}

1. Programa de Pós-Graduação em Ecologia, Conservação e Manejo da Vida Silvestre. 2. Laboratório de Ecologia Evolutiva e Biodiversidade, Departamento de Biologia Geral, ICB, Universidade Federal de Minas Gerais, Brasil. 3. Departamento de Biodiversidade, Evolução e Meio Ambiente, ICEB, Universidade Federal de Ouro Preto, Brasil. 4. Laboratório de Ecologia Evolutiva de Insetos de Dossel e Sucessão Natural.

\begin{abstract}
Resumo. As plantas possuem eficientes mecanismos a fim de se estabelecerem e permanecerem no ambiente. Um desses mecanismo é capacidade de rebrotamento que algumas espécies apresentam, muitas delas associadas à ambientes propensos à queimadas como é o caso dos ambientes dos campos rupestres. Nesse trabalho foi avaliado a capacidade e morfologia de rebrotamento pós distúrbio em duas populações típicas nos campos rupestres da Serra do Cipó, testando a hipótese de que o fogo (agente do distúrbio) é um agente seletivo dessas espécies e que elas apresentam então esse mecanismo de rebrotamento pós-distúrbio. Para o trabalho foram escolhidas as populações de Dalbergia miscolobium e de Vochysia thyrsoideae que perderam biomassa sobre o solo devido à intensa queimada na Serra do Cipó. Essas espécies apresentaram portanto, diferentes estratégias de rebrotamento onde D. miscolobium rebrotou a partir do chão, de estruturas capazes de armazenar amido (lignotuber) que favorecem esse tipo de recomposição de biomassa. Por outro lado, $V$. thyrsoidea garante a proteção de suas gemas pela espessura do CAP. Essas gemas protegidas passam a rebrotar a partir da casca da árvores, chamadas de gemas epicórmicas. Nossos dados além de mostrar o sucesso de recuperação dos órgãos fotossintéticos a partir do rebrotamento e das características da arquitetura de construção dos módulos para cada espécie, nos permitiu sugerir que o sucesso do rebrotamento e recuperação da biomassa sobre o solo, esteja relacionado com a quantidade de recursos alocados para essa função. $\mathrm{O}$ fogo portanto, pode atuar como agente seletivo de espécies eficientes quanto ao rebrotamento nos campos rupestres, uma vez que as características de rebrotamento exibidas para esse ambiente é similar às características apresentadas por outras espécies em outros ambientes também propensos à queimadas.
\end{abstract}

[Palavras chave: arquitetura de árvores, biomassa, brotamento vegetativo, Cerrado, Dalbergia miscolobium, fogo, Serra do Cipó, Vochysia thyrsoidea]

\begin{abstract}
Aвstract. Evaluation of resprout capacity pos disturbance in typical wood plants of campo rupestre grasslands: Plants have developed efficient mechanisms for establishing themselves and remaining in the environment. One such mechanism is the sprouting ability some species present, many of them associated to fire prone environments such as the campo rupestre grasslands. In this paper, we evaluated the ability and morphology of plants to re-sprout after disturbance in two typical populations of the campo rupestre grassland at Serra do Cipó, testing the hypothesis that fire (disturbance agent) is a selective agent for those species, and that in response they have developed the re-sprouting-after-disturbance mechanism. For the study, we selected populations of Dalbergia miscolobium and Vochysia thyrsoidea who had lost biomass on the ground due to the recurrent intense burning at Serra do Cipó. The two species showed different resprouting strategies. $D$. miscolobium resprouted from the ground, from structures capable of storing starch (lignotubers) that favours this kind of biomass rebuilding. $V$. thyrsoidea, on the other hand, ensures protection of its gems through the thickening of the CAP. These gems are protected to sprout from the barck of trees, and are called epicormic gems. Besides showing the successful recovery of the photosynthetic organs from re-sprouting and the characteristics of the architecture construction of modules for each species, our data has allowed us to suggest that the success of regrowth and recovery of biomass on the ground is related to the amount of resources allocated for this function. Therefore, fire can act as a selective agent for species with efficient re-sprouting mechanisms at the campos rupestre grasslands, since the characteristics of that environment for regrowth, illustrated in this study, are similar to features shown in other species of distinct fire prone environments.
\end{abstract}

[Keywords: biomass allocation, Dalbergia miscolobium, Cerrado, fire, Serra do Cipó, tree architecture, vegetative sprouting, Vochysia thyrsoidea] 


\section{INTRODUÇÃO}

As plantas exibem notável capacidade de se recomporem e se reestabelecerem, permanecendo no ambiente por meio de diversos mecanismos. Um deles é a capacidade de rebrotar os módulos da sua arquitetura, permitindo a recomposição da biomassa perdida durante algum distúrbio (Hallé et al. 1978; Bellingham \& Sparrow 2000). A recomposição vegetativa em ambientes que sofreram algum tipo de perturbação pode ser avaliada do ponto de vista da capacidade regenerativa da comunidade florística do local (Bond \& Midgley 2001).

O fogo é um processo ecológico antigo e característico de muitos ecossistemas terrestres, considerado um dos distúrbios mais dramático e comum, caracterizado como processo fundamentalem muitas comunidades ecológicas por todo o mundo (Bond \& Keeley 2005). Isso porque as queimadas são um fator de manutenção das estruturas das plantas na comunidade e as mudanças no regime do fogo podem alterar radicalmente a composição da comunidade (Lehmann et al. 2008). Alguns autores tem considerado o papel do fogo como uma força evolutiva que vem modelando as adaptações das plantas nos ambientes propensos à queimadas (Pausas et al. 2004).

Os campos rupestres compõem uma das muitas fitofisionomias que o bioma Cerrado (Brazilian savanna) apresenta. Ocorrem geralmente em altitudes acima de 900 metros e constituem comunidades herbáceo-arbustivas altamente adaptadas (Porembski 2007) formando diferentes habitats em função da topografia, natureza do substrato, profundidade do solo e microclima (Conceição et al. 2007; Medina \& Fernandes 2007). As áreas de campos rupestres são propensas às queimadas anuais (Ribeiro \& Figueira 2011) e como em muitas outras áreas do globo terrestre, o fogo tem um papel muito importante na ecologia e dinâmica de florescimento de algumas espécies (Neves \& Conceição 2010). Embora outras espécies não acionem o gatilho do florescimento pós passagem do fogo, elas recuperam sua biomassa por meio do rebrotamento dos meristemas axilares ou de raiz (Burrows 2002). Para serem capazes de rebrotar após distúrbio, as espécies arbóreas necessitam de meristemas axilares que sobreviveram a passagem do fogo e estoque de reserva para suportar seu crescimento (Bellingham \& Sparrow 2000).
Os estudos em relação a recuperação da biomassa sobre o solo como resultado de rebrotamento são realizados muitocomumente para Austrália, na região mediterrânea, Califórnia e África do Sul (Schwilk \& Keeley 2006; Hernández et al. 2011; Lamont \& Downes 2011). Essas regiões compreendem muitas áreas propensas às queimadas. Entretanto, para o Cerrado, região também propensa a queimadas, pouco se conhece a respeito da capacidade de recuperação a partir de estratégias de rebrotamento das espécies. Em Setembro de 2011 houve uma grande queimada em áreas de campos rupestres na Serra do Cipó e após o distúrbio obtivemos um cenário propício para estudar a capacidade de recuperação da biomassa sobre o solo das espécies dessa comunidade e comparar a estrutura de populações pós-fogo. Este trabalho se propôs avaliar as estratégias de recuperação do estrato arbóreo pós passagem do fogo em populações de duas espécies comuns e representativas no campo rupestre da Serra do Cipó. De modo que, espera-se que essas duas espécies apresentem propriedades ecomorfológicas que garantam o sucesso do rebrotamento pós queimada (Pausas et al. 2006; Lamont et al. 2011).

\section{MÉtodos}

\section{Área de estudo}

Nossa área de estudo está localizada na porção sul da Cadeia do Espinhaço, a 100km aproximadamente da cidade de Belo Horizonte, Brasil. A área está dentro da Reserva Natural Vellozia, na Serra do

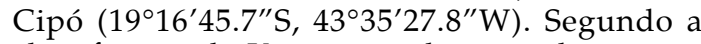
classificação de Köppen, o clima predominante é o tropical de altitude do tipo $\mathrm{Cwb}$, com verões quentes chuvosos e invernos secos. Madeira \& Fernandes (1999) reconhecem quatro períodos estacionais bem definidos: a estação seca (maiosetembro); estação pós-seca (outubro); estação chuvosa (novembro - janeiro) e estação pós chuvosa (fevereiro-abril). O estudo foi realizado a um mês após findada a queimada de causa natural na área, em Outubro de 2011. A queimada que ocorreu nos campos rupestres da Serra do Cipó deste ano foi considerada intensa, por ter atingido e destruído a copa de espécies arbustivas neste ambiente e também por ter queimado o substrato que fica alocado entre as rochas expostas.

\section{Espécies estudadas}

Dalbergia miscolobium Benth. (Fabaceae) - árvore conhecida popularmente como "jacarandá-docerrado". É uma espécie típica e reconhecida na região de cerrado por seu valor econômico, devido 
a qualidade de sua madeira, que é usada muito comumente em construção de casas, cercas e como carvão. Também apresenta alto potencial de uso em recuperação de áreas degradadas, visando a recomposição arbórea (Lorenzi 2000) Normalmente seu florescimento ocorre após a maturação da folhas durante a estação chuvosa e a perda de folhas e dispersão dos frutos ocorre no final da estação seca (Lemos Filho \& Isaias 2004).

Vochysia thyrsoidea Pohl. (Vochysiaceae) - popularmente conhecida como "gomeira" e "vinheiro do campo" por exsudar uma goma semelhante à goma arábica (Paviani \& Haridasan 1988). É uma planta com capacidade de acumular alumínio em seus tecidos e apresenta baixo desempenho na ausência desse elemento. Essa característica é importante no seu estabelecimento em solos distróficos, limitando sua distribuição e permitindo sua dominância nesse ambiente (Haridasan 2000; Campos et al. 2011). Seu período de floração vai de janeiro a abril e de setembro a dezembro. Seu período de frutificação ocorre de janeiro a novembro. Wagner et al. (2008) sugere que as gomas de $V$. thyrsoidea podem apresentar potencialidades como agente coagulante/antitrombótico devido a sua alta solubilidade em água e grande relação de agrupamentos ácidos. Os estudos ainda não avançaram nessa área e $V$. thyrsoidea ainda permanece como riqueza potencial como proposto por Matos Filho \& Rizzini (1960).

\section{Coleta de dados}

Dois afloramentos rochosos foram estudados. Um com dominância de D. miscolobium e o outro com dominância de $V$. thyrsoidea. A escolha dos indivíduos foi feita com a ajuda de uma bússola utilizando o método do vizinho mais próximo. Encontrado o primeiro indivíduo, nos orientamos pela bússola para a direção Leste (E) até o vizinho mais próximo. Este era marcado e nele realizadas as medidas. Assim sucederam as medidas em cada uma das espécies.

Para avaliar a capacidade de recuperação da copa das populações de D. miscolobium e $V$. thyrsoidea foram definidas algumas características da arquitetura a serem medidas (ver abaixo). Algumas das características de arquitetura escolhidas foram diferentes entre espécies. Isso se deve às diferenças de arquitetura das plantas e processos de construção de copa (Hallé et al. 1978). Essas medidas de arquitetura foram escolhidas pois representam diretamente a reconstituição da biomassa sobre o solo.

Para a população de D. miscolobium, foram selecionados doze indivíduos que tiveram sua biomassa sobre o solo totalmente queimada e doze indivíduos que não foram queimados. Nos próprios indivíduos totalmente queimados e não queimados medimos: altura total (m), CAP (circunferência do tronco a $1.30 \mathrm{~m}$ acima do chão, em $\mathrm{cm}$ ) e número de rebrotos (unidades). Os indivíduos totalmente queimados apresentaram uma estratégia de rebrotamento a partir do chão (resprouting basal stem), esse fato nos permitiu medir no maior ramo rebrotado: circunferência do ramo $(\mathrm{cm})$, comprimento $(\mathrm{cm})$, número de internós (unidade), o número de folhas produzidas (unidade) e grau de angulação (graus) do rebroto, enquanto que para os indivíduos não queimados de D. miscolobium, fizemos uma média do CAP $(\mathrm{cm})$, comprimento (cm), número de internós (unidade), o número de folhas produzidas (unidade) e grau de angulação (graus) de quatro rebrotos de cada indivíduo, uma vez que esses não queimados não apresentaram rebrotamento basal, mas sim rebrotos em suas copas.

Para a população de $V$. thyrsoidea foram também escolhidos doze indivíduos que foram queimados e doze que não foram queimados. Em todos esses indivíduos foram medidos: altura total $(\mathrm{m})$, altura do fuste $(\mathrm{m})$, CAP $(\mathrm{cm})$, a angulação dos pecíolos foliares (graus) e a distância média entre os internós para cada rebroto da planta $(\mathrm{cm})$. Vochysia thyrsoidea não exibiu rebrotamento na base do tronco queimado, como D. miscolobium. Por isso foram realizadas medidas de angulação dos pecíolos foliares e a distância média entre os internós a fim de detectar a capacidade de rebrotamento por gemas axilares.

\section{Análises estatísticas}

Análise de normalidade e homocedasticidade foram testadas usando os testes de KolmogorovSmirnov e Levene respectivamente. A capacidade de recuperação da copa das duas populações foi testada usando teste-t onde as variáveis dependentes foram as características de arquitetura e as variáveis independentes foram indivíduos queimados e não queimados.

Para verificar o potencial de rebrotamento, foi avaliado se indivíduos queimados e não queimados mais altos (variável independente) produzem maior quantidade de rebrotos (dependente) com Regressão Linear Simples e para avaliar quantos rebrotos são gerados em relação a altura (= número de rebrotos/altura total) entre as duas categorias queimados e não queimados, foi realizado um testet. Essas análises só foram possíveis para a população de D. miscolobium onde houve comportamento de rebrotamento. Todas as análises foram realizadas no software STATISTICA 7.

\section{Resultados}

\section{Dalbergia miscolobium}

Os indivíduos que tiveram sua biomassa sobre o solo totalmente queimada eram em média menores em relação aos indivíduos não queimados, apresentaram CAP duas vezes menor em relação aos não queimados 
Tabela 1. Comparação dos parâmetros de arquitetura entre os indivíduos queimados e não queimados de Dalbergia miscolobium e Vochysia thyrsoidea. ${ }^{*}$ p-valor $<0.05$.

Table 1. Comparison of parameters of architecture between burnt and unburned individuals Dalbergia miscolobium and Vochysia thyrsoidea. ${ }^{*}$ p-value $<0.05$.

\begin{tabular}{|c|c|c|}
\hline \multirow[t]{3}{*}{ Parâmetros } & \multicolumn{2}{|c|}{ Dalbergia miscolobium } \\
\hline & \multicolumn{2}{|c|}{ Indivíduos } \\
\hline & Queimados & $\begin{array}{c}\text { Não } \\
\text { queimados }\end{array}$ \\
\hline Altura total $(\mathrm{m})^{*}$ & $0.98 \pm 0.22$ & $1.99 \pm 0.33$ \\
\hline $\mathrm{CAP}(\mathrm{cm})^{*}$ & $7 \pm 4.39$ & $15.5 \pm 6.19$ \\
\hline Número de rebrotos (und)* & $6.16 \pm 2.16$ & $4 \pm 1.98$ \\
\hline \multirow{3}{*}{$\begin{array}{l}\text { Número de rebrotos/altura } \\
\text { total }^{*}\end{array}$} & $6.7 \pm 3.16$ & $1.98 \pm 0.63$ \\
\hline & \multicolumn{2}{|c|}{ Rebrotos } \\
\hline & Queimados & $\begin{array}{c}\text { Não } \\
\text { queimados }\end{array}$ \\
\hline Circunferência do ramo $(\mathrm{cm})^{*}$ & $3.15 \pm 1.11$ & $1.52 \pm 0.65$ \\
\hline Comprimento $(\mathrm{cm})^{*}$ & $70.2 \pm 17.07$ & $41.95 \pm 14.03$ \\
\hline Número de internós (und)* & $18 \pm 3.1$ & $11.7 \pm 4.17$ \\
\hline Grau de angulação $\left({ }^{\circ}\right)^{*}$ & $17.08 \pm 13.04$ & $29.5 \pm 12.5$ \\
\hline \multirow[t]{3}{*}{ Número de folhas (und) } & $41.1 \pm 28.8$ & $23.04 \pm 12.3$ \\
\hline & \multicolumn{2}{|c|}{ Vochysia thyrsoidea } \\
\hline & Queimados & $\begin{array}{c}\text { Não } \\
\text { queimados }\end{array}$ \\
\hline Altura total (m) & $1.85 \pm 0.57$ & $1.81 \pm 0.62$ \\
\hline Altura do fuste (m) & $0.66 \pm 0.32$ & $0.73 \pm 0.3$ \\
\hline CAP $(\mathrm{cm})$ & $0.3 \pm 0.07$ & $0.27 \pm 0.13$ \\
\hline $\begin{array}{l}\text { Angulação dos pecíolos } \\
\text { foliares }\left({ }^{\circ}\right)\end{array}$ & s $34.3 \pm 7.8$ & $39.6 \pm 9.77$ \\
\hline $\begin{array}{l}\text { Distância média entre os } \\
\text { internós }(\mathrm{cm})^{*}\end{array}$ & s $12.8 \pm 4.98$ & $22.9 \pm 6.83$ \\
\hline
\end{tabular}

e tiveram maior quantidade de rebrotos comparado aos não queimados (Tabela 1).

O número de rebrotos não diferiru entre os indivíduos maiores e menores de D. miscolobium. (Regressão Linear Simples: $\mathrm{F}_{(1,22)}=2.44 ; \mathrm{r}=0.31$; $P>0.05)$, entretanto, os indivíduos queimados mostraram que em relação à sua própria altura eles investem em uma quantidade maior de rebrotos quando comparado aos não queimados (Tabela 1).

Osramos rebrotadosdosindivíduosqueimados tiveram circunferência do ramo duas vezes maior em relação aos rebrotos dos indivíduos não queimados (Tabela 1), os rebrotos dos indivíduos queimados tiveram ramos com quase o dobro do comprimento em relação aos ramos dos indivíduos não queimados (Tabela 1), maior quantidade de número de internós (Tabela 1) e apresentaram menor angulação (Tabela 1). Entretanto não houve diferença para o número de folhas nos ramos rebrotados entre indivíduos queimados e não queimados (Tabela 1).
Os indivíduos queimados de D. miscolobium foram aqueles indivíduos menores e de CAP mais fino. Entretanto, esses indivíduos apresentaram capacidade de rebrotamento maior em relação aos não queimados. Esses indivíduos que foram queimados usam da estratégia de rebrotamento basal (Burrows 2002), enquanto que os indivíduos não queimados rebrotaram nos galhos que permanecem vivos na copa. Esses rebrotos por sua vez mostraram várias diferenças quanto a velocidade na qual recuperam sua biomassa. No mesmo período de tempo, os rebrotos dos indivíduos queimados tiveram maior comprimento, CAP e quantidade de internós. Mesmo a quantidade de folhas não sendo significativamente diferente, elas foram quase o dobro nos indivíduos que foram queimados.

\section{Vochysia thyrsoidea}

Os indivíduos queimados e não queimados de $V$. thyrsoidea não apresentaram diferenças quanto altura total (Tabela 1); quanto altura do fuste (Tabela 1) e em relação ao CAP (Tabela 1). A angulação dos pecíolos foliares também não apresentou variação evidente (Tabela 1), entretanto, a distância média entre os internós para os indivíduos queimados foi menor em relação a distância entre um internó e outro para os indivíduos não queimados (Tabela 1). Ou seja, as duas categorias avaliadas de queimados e não queimados para a população de $V$. thysoidea têm as mesmas características quanto aos aspectos de arquitetura sobre o solo. Entretanto os indivíduos que sofreram o dano causado pelo fogo exibem suas folhas em um tempo mais curto, medido pela distância média entre um internó e outro; em relação aos indivíduos que não foram queimados.

\section{DISCUSSÃO}

Os resultados mostraram que embora as estratégias de rebrotamento encontradas para D. miscolobium e $V$. thyrsoidea sejam diferentes, as duas populações recuperam sua biomassa perdida. Os indivíduos de $D$. miscolobium que foram queimados rebrotaram a partir de estoque de amido na raiz. As estruturas que armazenam esse amido é chamada "lignotuber" que é resultado de uma protuberância na raiz que a protege de ser destruída quando o fogo queima o tronco/ haste da planta (Canadell \& Lopez-Soria 1998). Em função dessa característica, muitas plantas, 
como no caso de D. miscolobium crescem com o caule múltiplo, decorrente do "lignotuber" (Molinas \& Verdaguer 1993). No caso de $V$. thyrsoidea seu modelo de rebrotamento foi a partir das gemas epicórmicas, que se localizam ao longo do tronco e de gemas axilares, apresentando característica de rebrotamento similar a de eucalipto mostrado no trabalho de Lawes et al. (2011) e também de algumas árvores do Mediterrâneo, que também exibem cascas grossas e e brotação epicórmica após distúrbio causado pelo fogo (Dantas et al. 2013). A espessura da casca das árvores é uma estratégia que garante proteção efetiva à planta, quando seus ramos são totalmente queimados, por proteger as gemas laterais garantindo o rebrotamento epicórmico (Dantas et al. 2013). Esses resultados corroboram nossa hipótese de que essas estratégias foram selecionadas pelo agente fogo em função da sua capacidade de rebrotar logo após distúrbio.

As duas populações exibiram notável capacidade de recuperação dos órgãos fotossintéticos. Segundo Moreira et al. (2012) a sobrevivência pós-rebrotamento não se relaciona diretamente ao estado pré-distúrbio da planta, mas à quantidade de biomassa produzida. Isso sugere que para as plantas capazes de rebrotar vivendo em ambientes propensos à queimadas, a capacidade fotossintética dos rebrotos devem estar sob forte pressão seletiva para ser altamente eficientes fotossinteticamente. As novas folhas tendem a ser maiores, com maior massa seca, maior quantidade de clorofila e consequentemente, serem mais eficientes na fotossíntese. Neste trabalho, verificamos que os novos rebrotos crescem mais, mantêm uma angulação pequena que favorece o crescimento na vertical e exibem pouca distância entre um internó e outro, apontando então essa eficiência fotossintética para essas populações.

Em seu trabalho, Boucher et al. (1994) mostrou que Vochysia ferruginea é uma espécie resiliente pós-distúrbio na Floresta Tropical da Nicarágua, exibindo mudanças substanciais em relação aos valores das variáveis/características no pré-distúrbio. Entretanto, no nosso trabalho, $V$. thyrsoidea se mostra como uma espécie resistente, exibindo poucas diferenças em relação suas variáveis no pré-distúrbio, enquanto D. miscolobium se mostra mais resiliente nos campos rupestres. Outra diferença também encontrada no nosso trabalho é em relação à influência do tamanho da planta no vigor do rebrotamento. Moreira et al. (2012) mostrou que plantas maiores rebrotam mais vigorosamente em relação às plantas menores. Nossos resultados mostraram que o vigor de rebrotamento independe da altura da planta, sugerindo que talvez o vigor de rebrotamento esteja mais relacionado à quantidade de recursos estocados e a capacidade de mobilização destes para a recuperação da parte aérea (Canadell \& Lopez-Soria 1998).

Este trabalho nos permite concluir que para as duas espécies escolhidas para avaliar a capacidade de rebrotamento, o fogo pode ser considerado um fator de seleção de espécies com capacidade de rebrotamento pós-distúrbio nos campos rupestres; e a compreensão dos diferentes mecanismos de persistência dessas plantas nos permite fazer implicações a respeito da sobrevivência dessas espécies frente a frequência com que o distúrbio ocorre nesse ambiente.

\section{REFERÊNCIAS}

BeLlinghan, PJ \& AD Sparrow. 2000. Resprouting as a life history strategy in woody plant communities. Oikos, 89: 409-416.

BOND, WJ \& JE KEELEY. 2005. Fire as a global 'herbivore': the ecology and evolution of flammable ecosystems. Trends Ecol Evol, 20:387-394.

Bond, WJ \& JJ Midgley. 2001. Ecology of sprouting in woody plants: the persistence niche. Trends Ecol Evol, 16:45-51.

BOUChER, DH; JH VANDERMEer; MA Mallona; N ZAMORA \& I PERFECTO. 1994. Resistance and resilience in a directly regenerating rainforest:Nicaraguan trees of the Vochysiaceae after Hurricane Joan. Forest Ecol Manag, 68:127-136.

Burrows, GE. 2002. Epicormic strand structure in Angophora, Eucalyptus and Lophostemon (Myrtaceae): implications for fore resistance and recovery. New Phytol, 153:111-131.

Campos, HR; SA Cardoso Silva \& FJ Soares Junior. 2011. Estrutura populacional de Vochysia thyrsoidea Pohl. em uma área de transição entre Cerrado stricto sensu e campo rupestre, no município de Ingá, Minas Gerais, Brasil. Rev Bio, 17:5-14.

CANADELL, J \& L LÓPEZ-SORIA 1998. Lignotuber reserves support regrowth following clipping of two Mediterranean shrubs. Funct Ecol, 12:31-38.

Conceição, AA; JR Pirani \& ST Meirelles. 2007. Floristics, structure and soil of insular vegetation in four quartzitesandstone outcrops of "Chapada Diamantina", Northeast Brazil. Rev Bras Bot, 30:641-656.

Dantas, V; MA Batalha \& JG Pausas. 2013. Fire drives functional thresholds on the savanna-forest transition. Ecology, 94:2454-2463.

Hallé, F; RAA Oldeman \& PB Tomlinson. 1978. Tropical trees and forests. Springer-Verlag. Berlin. 310 pp.

HARIDASAN, M. 2000. Nutrição mineral de plantas nativas do cerrado. R Bras Fisiol Veg, 12:54-64. 
Hernández, EI; JG Pausas \& A Vilagrosa. 2011. Leaf physiological traits in relation to resprouter ability in the Mediterranean Basin. Plant Ecol, 212:1959-1966.

LAMONT, BB \& KS DOWNES. 2011. Fire-stimulated flowering among resprouters and geophytes in Australia and South Africa. Plant Ecol, 212:2111-2125.

Lamont, BB; NJ Enright \& T He. 2011. Fitness and evolution of resprouters in relation to fire. Plant Ecol, 212:1945-1957.

Lawes, MJ; A Richards; J Dathe \& JJ Midgley. 2011. Barck thickness determines fire resistance of selected tree species from frire-prone tropical savana in North Australia. Plant Ecol, 212:2057-2069.

LEHMANN, CER; LD PRIOR; RJ WILLIAMS \& DMJS BOWMAN. 2008. Spatio-temporal trends in tree cover of a tropical mesic savanna are driven by landscape disturbance. J Appl Ecol, 45:1304-1311.

Lemos Filho, JP \& RMS Isaias. 2004. Comparative stomatal condutance and chlorophyll a fluorescence in leaves vs. fruits of the cerrado legume tree, Dalbergia miscolobium. Braz J Plant Physiol, 16:89-93.

LoRENZI, H. 2000. Árvores Brasileiras: Manual de Identificação e Cultivo de Plantas Arbóreas Nativas do Brasil. 2da ed. Instituto Plantarum, Nova Odessa, SP, Brasil. 384 pp.

MADEIRA, JA \& GW FERNANDES. 1999. Reprodutive phenology o sympatric taxa of Chamaecrista (Leguminosae) in Serra do Cipó, Brazil. J Trop Ecol, 15:463-479.

Matos Filho, A \& CT Rizzini. 1960. Contribuição ao estudo de Vochysia thyrsoidea POHL. (Vochysiaceae). Rodriguésia, 23/24(35/36):83-98.

Medina, BMO \& GW Fernandes. 2007. The potential of natural regeneration of rocky outcrop vegetation on rupestrian field soils in "Serra do Cipó", Brazil. Rev Bras Bot, 30:665-678.
MOLINAS, ML \& D VERDAGUER. 1993. Lignotuber ontogeny in the cork-oak (Quercus suber; Fabaceae). Am J Bot, 80: 182-191.

Moreira, B; J Tormo \& JG Pausas. 2012. To resprout or not to resprout: factors driving intraspecific variability in resprout. Oikos, 121:1577-1584.

Neves, SPS \& AA ConceiçÃo. 2010. Campo rupestre recém-queimado na Chapada Diamantina, Bahia, Brasil: plantas de rebrota e sementes, com espécies endêmicas na rocha. Acta Bot Bras, 24:697-707.

Pausas, JG; JE Keeley \& M Verdú. 2006. Inferring differential evolutionary processes of plant persistence traits in Northern Hemisphere Mediterranean fire-prone ecosystems. J Ecol, 94:31-39.

Pausas, JG; RA Bradstock; DA Keith; JE Keeley \& GCTE FIRE NETWORK. 2004. Plant functional traits in relation to fire in crown-fire ecosystems. Ecology, 85:1085-1100.

Paviani, TI \& M Haridasan. 1988. Tuberosidade em Vochysia thyrsoidea Pohl. Ciência e Cultura, 40:998-1003.

Porembski, S. 2007. Tropical inselbergs: habitat types, adaptive strategies and diversity patterns. Rev Bras Bot, 30:579-586.

Ribeiro, MC \& JEC Figueira. 2011. Uma abordagem histórica do fogo no Parque Nacional da Serra do Cipó, Minas Gerais - Brasil. Biodiversidade Brasileira, 1: 212-227.

SCWILK, DW \& JE KEELEY. 2006. The role of fire refugia in the distribution of Pinus sabiniana (Pinaceae) in the southern Sierra Nevada. Madroño, 53:364-372.

WAGNER, R; FF SiMAS; GL SASSAKI; M IACOMINI; MA Silva \& PAJ Gorin. 2008. A high-viscosity glycoglucuronomannan from the gum exudate of Vochysia thyrsoidea: Comparison with those of other Vochysia spp. Carbohyd Polym, 72:382-389. 